\title{
Distributed Resource Allocation for D2DCommunication Networks Based in Game Theory
}

\author{
F. W. Zaki ${ }^{1}$, S. Kishk ${ }^{1}$, N. H. Almofari ${ }^{(1,2)}$ \\ ${ }^{1}$ Communication and Electronics Dept. Faculty of Engineering, Mansoura University, Egypt. \\ ${ }^{2}$ Ministry of Higher Education and Scientific Research, Yemen.
}

\begin{abstract}
The next generation expected to provide high data rate to handle the huge demand on new dataconsuming applications and services, Also the higher number of connected devices due to using new technologies such as internet of things (IoT) will make the challenge more difficult. New techniques must be used to face this challenges such as base station densification, use new high frequency bands, Machine to Machine (M2M) and Device to Device (D2D) communication. In heterogeneous multitier networks, D2D communication is used as underlay tier to enhance the system spectral efficiency and increase the data rate. This complicate the resource allocation process in this networks. Distributed algorithms is used to solve the resource allocation problems with low computational complexity.
\end{abstract}

In this paper, the allocation problem is formulated to maximize the system data rate while confiding the cochannel interference resulted from the D2D underlay tier which share the same resources with the cellular users. Auction based algorithm is used to solve the proposed allocation problem in distributed manner with very low complexity compared to the centralized methods.

Keywords: Resource allocation, Relay assisted D2D, Auction.

\section{INTRODUCTION}

The increased demand on wireless networks services and higher data rates in next generation networks need new technologies and algorithms to handle this requirement. D2D communications is one of the LTE-A technologies, where D2D pairs used as underlay of the cellular network to increase the efficiency of using radio spectrum [1]. In D2D communication, D2D pairs communicate directly using cellular resource without assistance of eNB. This increase resources utilization where the same resource can be used simultaneously by the cellular UE and D2D pair (Reuse gain). Also the short path between the D2D user equipment increase the link efficiency (a proximity gain). D2D pairs use one link resource instead of using resource for uplink and downlink resources in the case of cellular mode (hop gain)[2].

D2D communication can be categorized in to two types depend on the spectrum used by the D2D pairs: unlicensed spectrum is used by ad hoc and personal area networks in short range communications such as Bluetooth and Direct WiFi.licensed (cellular) spectrum is proposed recently to use by D2D pairs in cellular networks to enhance the spectral and energy efficiency [3].

D2D communication increase the spectral efficiency and improve the cellular coverage, but introduce interference to cellular users, which need to develop new efficient interference from underlay tier and realize the benefits of D2D communication [4]. The heterogeneous nature of the next generation networks, the base station densification, using small base station and relays, and using D2D communication undelaying cellular network make the resource allocation in these networks very complex. High computational complexity of the centralized solution of the resource allocation problem prompted the researchers to find distributed methods with lower complexity.

New methods coming from economic such as Auction, stable matching and message passing and other methods such as Stackelberg game gain attention as a game theory based approach to solve the complex resource allocation problems[5].

Authors of [6] analyze the D2D communication as underlay tier of the cellular UEs and propose a power control scheme to restrict the interference between the cellular link and the D2D link aiming to increase the overall data rate. In [7] the resource of cellular uplink is shared with the D2D users and 


\section{F. W. Zaki et al.}

interference tracing approach is used to mitigate the interference from cellular UEs to D2D UEs, while interference broadcasting method is used to address the interference introduced from D2D to the cellular link. In [8], the resource allocation problem of 3GPP-LTE network with D2D UEs in the underlay tier is formulated as Mixed Integer Nonlinear Program (MINP) which is very complex to solve in the small scheduling duration in the LTE networks. Authors proposed a greedy heuristic algorithm to solve this allocation problem and reduce the interference effects on the cellular users.

To reduce co-channel interference in small cell networks, authors in [9] use distributed algorithm to implement efficient resource allocation in the downlink. They use an algorithm based in pricing concept, where each base station choose initial power profile and scheduling weights, calculate the prices, and update the power profile and schedule weights iteratively to improve the data rate and fairness of sharing resources among users. In [10] D2D communication are used as underlay to improve the system performance and a reverse iterative combinatorial auction mechanism is used for resource allocation.

In reverse iterative combinatorial auction the resources are considered as the bidders and the D2D links as the objects. This allocation mechanism reduces the complexity and also reduces the effect of interference on the macro users. A similar work based on combinatorial auction is introduced in [11], [12].

Stable matching based algorithm is proposed by authors of [13] to satisfy QoS requirements for underlay D2D pairs while restrict the interference on the cellular user.

In this paper the resource allocation problem is formulated to maximize the data rate of cellular and D2D users share the same resource with considering the co-channel interference where the intercell interference can be mitigated using proper power control. Resource allocation algorithm based on auction game is proposed to efficiently share the resource of cellular users with D2D UEs.

The rest of this paper is organized as follow: Section (2) present the system model used in the paper including the channel model and the calculations of the Signal to Interference plus Noise Ratio (SINR). The optimization problem is introduced in section (3). In section (4) the concept of auction algorithms and it's usage to solve the resource allocation problem is explained, Also Auction based algorithm is introduced to solve the resource allocation problem distributively. The results of the matlab simulation is presented and discussed in section (5). Finally the paper is concluded in section (6).

\section{SySTEM MODEL}

In our previous work [14], system model of single cell two tier LTE-A network containing four energy harvesting relays is considered. The resource allocation problem is formulated to maximize the sum data rate with considering the inter cell interference using reference user concept, also the user association is implemented considering the available energy in the energy harvesting relays using survival probability concept.

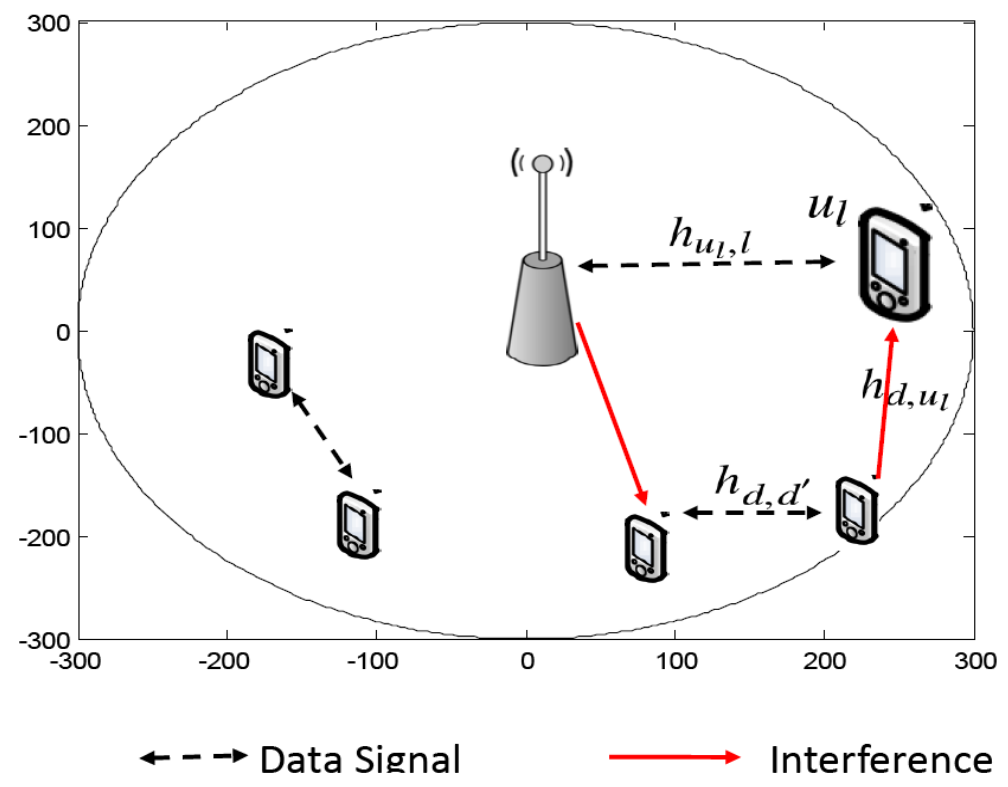

Figure1. System Model 
In this work one relay cell containing cellular and D2D users share the same resources is considered to investigate the effect ofintracell interference introduced from the D2D UEs to the cellular UEs. The Relay is located at the center of 300 meterradius cell. The cellular and D2D transmitters are distributed randomly in the cell area. The D2D receivers is distributed around the D2D transmitter in distance lower than 80 meterto realize the effectiveness of using direct D2D link. It is assumed that $N$ resource blocks are already assigned to the cellularUEs and the D2D users share the same resource blocks with the cellular UEs in undelaying manner. It is concentrated onthe effect of co-channel interference introduced from underlaytier on the cellular users.Figure1 illustrates the interferencerelations and the channel gains where $u_{l}$ is cellular user servedby layer 3 relay $l, h_{u_{l}, l}$ is the channel gain between the cellularuser $u_{l}$ and the relay $l, h_{d, u_{l}}$ is the channel gain between theD2D transmitter and cellular user $u_{l}$ and $h_{d, d}$ is the channelgain between the D2D pairs.

\subsection{Radio Resource and Channel Model}

The bandwidth of LTE system is divided into resource blocks (RBs) with equal size, where each RB occupies one time slot $(0.5 \mathrm{~ms})$ and $180 \mathrm{KHZ}$ in the frequency domain. The RBs separated from each other by $15 \mathrm{KHZ}$. LTE use OFDMA as multiple access technology in the downlink to benefit from its immunity against multipath delay and its high spectral efficiency, while using SC-OFDMA in the uplink due to its lower peak-to-average Power ratio (PAPR).

The cannel model introduced in [8] is used in this paper to estimate the pathloss and channel gains, where the pathloss between the relay 1 and UE is given by

$L_{d B}(d)=40\left(1-4 \times 10^{-3} h_{l}\right) \log _{10}(d)-18 \log _{10}\left(h_{l}\right)+21 \log _{10}\left(f_{c}\right)+80$

Where $d$ is the distance between the relay and UE in kilometer $(\mathrm{KM}), f_{c}$ is the carrier frequency in MHZ, and $h_{l}$ is the relay antenna height in meters.

The gain between $\mathrm{D} 2 \mathrm{D}$ pairs is given by

$h_{d, d^{\prime}}=K_{d, d^{\prime}} d_{d, d^{\prime}}^{-\alpha}$

Where $K_{d, d}$ the normalization constant chosen depending on the radio propagation environment, $d_{d, d^{\prime}}$ is the distance between the D2D pairs, and $\alpha$ is the pathloss exponent.

\subsection{SINR and Data Rate Calculations}

The Signal to Interference plus Noise (SINR) of the cellular and D2D users must be calculated and considered as important parameter to maximize the system data rate. The SINR of user $u_{l}$ can be given by (3)

$\gamma_{u_{l}}=\frac{P_{l} h_{u_{l}, l}}{I_{u_{l}}+N_{o}}$

Where $P_{l}$ is the transmission power of relay $l, N_{o}$ is the thermal noise and $I_{u_{l}}$ is the interference power received by user $u_{l}$ which is in our model coming from the group of D2D pairs sharing resources with the cellular user $u_{l} . I_{u_{l}}$ can be given by equation (4)

$I_{u_{l}}=\sum_{u_{d} \in D_{u_{l}}} P_{d} h_{d, u_{l}}$

Where $u_{d}$ is D2D UE and $D_{u_{l}}$ is a group of D2D pairs share the same resource with cellular user $u_{l}$. The data rate of the cellular user $u_{l}$ given by Shannon equation as

$R_{u_{l}}=B_{R B} \log _{2}\left(1+\frac{P_{l} h_{u_{l}, l}}{{\sum u_{d} \in D_{u_{l}} P_{d} h_{d, u_{l}}+N_{o}}_{1}}\right)$

In the same manner the data rate of the D2D user $u_{d}$ which share the same resource with the cellular user $u_{l}$ is given by equation (6)

$R_{u_{d}}=B_{R B} \log _{2}\left(1+\frac{P_{d} h_{d, d^{\prime}}}{P_{l} h_{l, d}+\sum_{u_{d}^{\prime} \in D_{u_{l}}-d} P_{d} h_{d, d^{\prime}}+N_{o}}\right)$

Where $P_{l} h_{l, d}$ represent the interference power from the relay $l$ and the term $\sum_{u_{d^{\prime}} \in D_{u_{l}}-d} P_{d} h_{d, d^{\prime}}$ is the interference from other D2D pairs share the same resource blocks with D2Duser $u_{d}$. It is assumed that, 
there is set of resource blocks $N=\{1,2,3, \ldots . N\}$ where each resource block assigned to one cellular user. The data rate achieved by the $n_{t h}$ resource block (Channel) is given by

$R_{n}=R_{u_{l}}+\sum_{u_{d} \in D_{u_{l}}} R_{u_{d}}$

and the downlink system data rate equal to

$R_{l}=\sum_{n=1}^{N}\left(R_{u_{l}}+\sum_{u_{d} \in D_{u_{l}}} R_{u_{d}}\right)$

\section{Problem Formulation}

It is assumed that each cellular user $u_{l}$ have one resource block already assigned to it (The number of resource blocks equal to the number of cellular users). Also, it is assumed that each D2D use one or more resource blocks and the resource block can be shared with one or more D2D pairs. The D2D pairs divided into groups, where $D$ is the set of $D 2 D$ pair groups $D=\{1,2, \ldots . K\}$. The data rate achieved when the $n_{t h}$ cellular user share resources with the members of the $k_{t h}$ group of D2D pairs can be defined as:

$$
\begin{aligned}
& R(n, k)=B_{R B} * \\
& \log _{2}\left(1+\frac{P_{l} h_{u_{l}(n), l}}{\sum_{u_{d} \in D_{u_{l}} P_{d} h_{d, u_{l}^{(n)}}^{(n)}+N_{o}}}\right)+\sum_{u_{d} \in D_{k}} \log _{2}\left(1+\frac{P_{d} h_{d, d^{\prime}}}{P_{l} h_{l, d}+\sum_{u_{d}} \in D_{u_{l}-d^{-}} P_{d} h_{d, d^{\prime}}+N_{o}}\right)
\end{aligned}
$$

The objective of the optimization problem is to maximize the system data rate by taking into account the co-channel interference to improve the system performance.

$\max \sum_{n=1}^{N} \sum_{k=1}^{K} a_{n}^{k} R(n, k)$

Subject to:

$P_{l} \leq P_{l}^{\max }$

$P_{d} \leq P_{d}^{\max }$

$\sum_{u_{d} \in D_{u_{l}}} P_{d} h_{d, u_{l}^{(n)}} \leq I^{(t h)} \forall n \in N^{\prime}, k \in \mathcal{D}$

$\sum_{n=1}^{n} a_{n}^{k} \leq 1 \quad \forall k \in \mathcal{D}$

$\sum_{k=1}^{K} a_{n}^{k} \leq 1 \quad \forall k \in \mathcal{D}$

$a_{n}^{k} \in\{0,1\} \forall n \in \hat{\mathrm{N}}, \forall k \in \mathrm{D}$

Where $a_{n}^{k}$ is the allocation indicator which equal to 1 if the $n_{t h}$ cellular user share resources with the members of the $k_{t h}$ group, and equal to 0 elsewhere. The constraint in (11) and (12) insure that the transmission power of relay and UE don't exceeds the maximum power. The constraint in (13) restrict the underlay interference to be lower than predefined threshold, and the constraint in 14 and 15 state that, each D2D group share resource with only one cellular user and vice versa. This constraint not contradict with our previous assumption because each group may contain more than one D2D pair and each $\mathrm{D} 2 \mathrm{D}$ pair can belong to more than one group.

\section{Auction Algorithms for Resource Allocation}

The resource allocation using auction concept use bidding procedure, where each agent (D2D transmitter) bid for resources (Resource Blocks RBs). The relay (or eNB) work as Auctioneer who assign resources to the highest bidder. The D2D transmitter (which need resource block) raise the bid for the required resource block depending on the net value (which is the benefit of using this resource - the cost paid) [15].

To understand the concept of auction, assume that each resource $j$ assign a cost $c_{j}$ and each agent iget a benefit $B_{i j}$ of using resource. The net value obtained by agent $i$ when use resource $j$ is equal to $B_{i j}-c_{j}$. The assignment of resources to agents aiming to maximize the net value

$B_{i j^{\prime}}-c_{j^{\prime}}=\max _{j}\left(B_{i j}-c_{j}\right)-\epsilon$ 
Equation (17) indicate that, agent $i$ must assigned to resource $j$ ' which give the maximum net value. $\epsilon$ is positive constant used to avoid loops due to unsatisfying equilibrium condition. Equation (17) called $\epsilon$-complementary slackness, when $\epsilon=0$ it is called equilibrium condition [16].

To solve the above optimization problem using auction game, assume the benefit to make the $k_{t h}$ D2D group share resource with the $n_{t h}$ cellular user equal to $R(n, k)$ and the cost paid is the interference as follows:

$c_{n}^{k}=I_{k}^{(n)}-I^{(t h)}$

Where $I_{k}^{(n)}$ is the interference caused by D2D members of the $k_{t h}$ group to the $n_{t h}$ cellular user and $I^{(t h)}$ is the interference threshold. The utility function defined as

$U_{n}^{k}=R(n, k)-\left(I_{k}^{(n)}-I^{(t h)}\right)$

Algorithm (1) below is used to implement the auction process with considering the interference constraint satisfactions. The utility matrix is constructed as follow

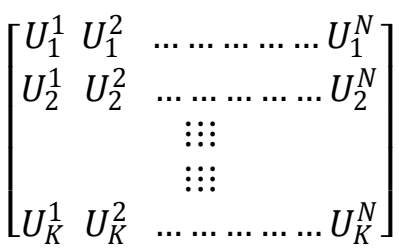

The D2D groups bids to get the maximum utility, the algorithm converge when the $\epsilon$-complementary slackness condition is satisfied for all agents (D2D groups). To get the utility matrix the estimated data rate for each assignment must be calculated using equation (9).

Algorithm 1: Resource Blocks Allocation Using Auction.

Input: Utility Matrix $U_{n}^{k}$

Output: RB allocation vector a.

Initialize $a:=0$

Repeat

- Choose unassigned D2D group k, calculate its maximum and second maximum utility as

$$
\begin{gathered}
\gamma_{\hat{n}}^{k}=\max _{n} U_{n}^{k} \\
\delta_{\hat{n}}^{k}=\max _{n, n \neq \mathrm{n}} U_{n}^{k}
\end{gathered}
$$

- Update the aggregated interference on resource block $\dot{n}$

$$
I^{(\hat{n})}=I^{(\text {ń) }}+I^{\text {(fromadding } \left.k_{\text {th }} \text { group }\right)}
$$

if $I^{(\mathrm{n})} \leq I^{(t h)}$ then

$a_{k}^{\text {ń }}=1$ assign resource block $n$ to the $k_{t h}$ D2D group

Update the cost as $c_{\hat{\mathrm{n}}}^{k}=c_{\hat{\mathrm{n}}}^{k}+\gamma_{\hat{\mathrm{n}}}^{k}-\delta_{\hat{\mathrm{n}}}^{k}+\epsilon$

end if

if resource block $n$ has been assigned to another D2D group $k$ before then

Remove that assignment

end if

if the D2D group $k$ had been assigned to a resource block $n$ prior to being assigned to $n$ then assign $k$ to $n$

\section{Until}

end if

All pairs satisfy equation

$$
R(\hat{n}, k)-c_{\text {ń }}=\max _{j}\left(R(n, k)-c_{n}\right)-\epsilon
$$

To calculate the distances between users and relay, the method introduced in [17] is used. The cell area is divided to 500 zone and 500 sector as shown in Figure (2) and each user is located in one resulted small area. The zone surface (C) can be obtain as $\frac{\pi r^{2}}{\text { NumberofZones }}$ and

$C=\pi r^{2}(z+1)-\pi r^{2}$

This gives the distance from any user to the relay as 
$r_{(z+1)}=\sqrt{\frac{C}{\pi}-r^{2}}$

Where $r_{0}=0$.

The distance between two D2D pairs can be obtained as

$d_{d, d^{\prime}}=r_{1}^{2}+r_{2}^{2}-2 r_{1} r_{2} \cos (\theta)$

Where $\theta$ equal to:

$\theta=\frac{2 \pi}{\text { NumberofSectors }}\left|d_{(S N)}-d_{(S N)}^{\prime}\right|$

whered $d_{(S N)}$ is the sector number of D2D pair d.

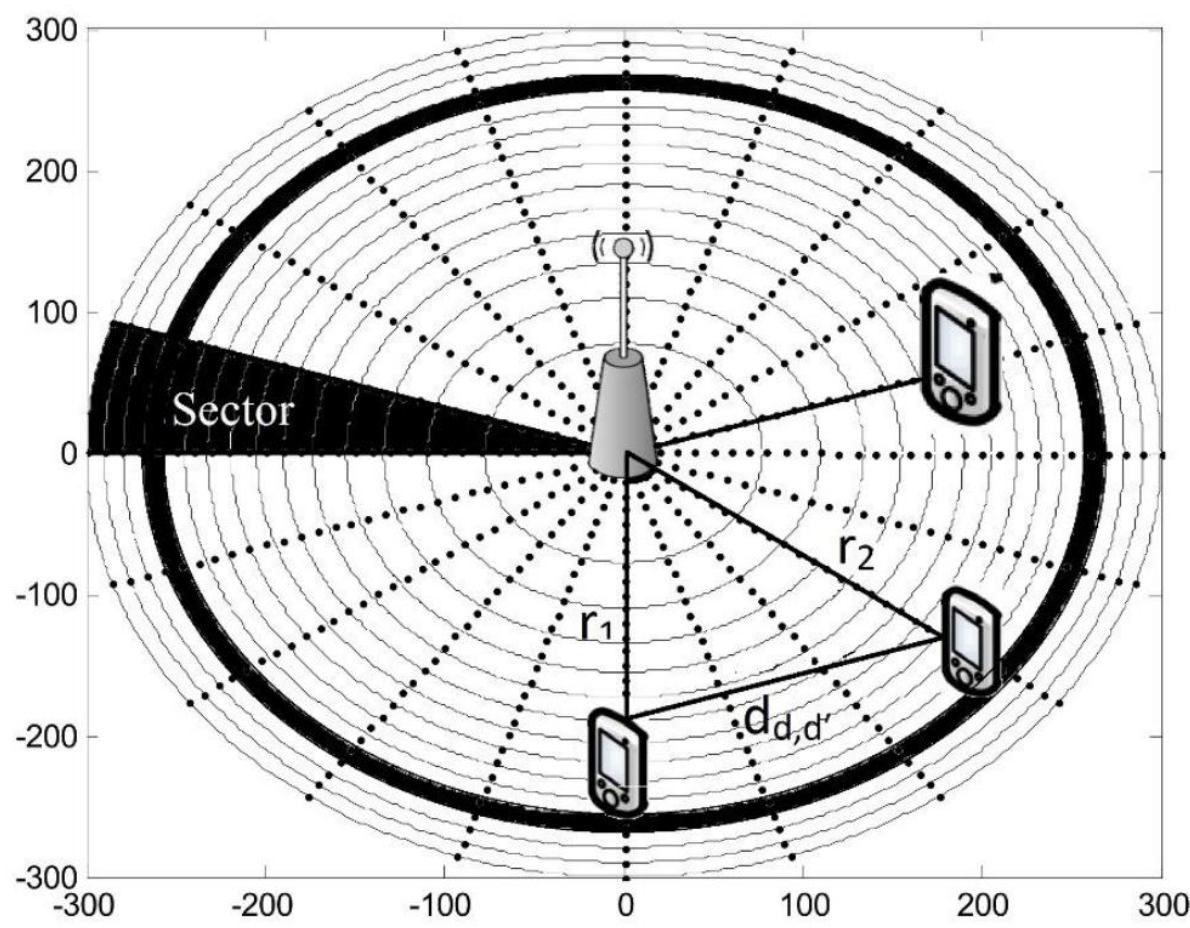

Figure2.Cell Dividing to Calculate distances.

\section{Simulation RESUltS AND DiscUSSION}

In a previous work, LTE-A macro cell with 4 relays was considered. In the present work, a relay cell with 300 meter radius is investigated to study the effect of co-channel interference when D2D users are considered as underlaying cellular users. The relay is located in the center of the 300 meter radius cell and cellular users and the D2D transmitters are distributed randomly around it. The D2D receiving ends are distributed around the D2D transmitters with maximum distance 80 meters. The channels are characterized by channel model presented in section (2.1). The network parameters are listed in Table (1). Each cellular user assigned one resource block while D2D don't have resource blocks for their won. They only share the resource with the cellular users do not interfere with them.

The number of cellular and D2D pairs is increased in each experiment to study the effect of increasing them on the system data rate.

Table1.Network Parameters

\begin{tabular}{|l|l|}
\hline Parameter & Value \\
\hline Cell radius & $300 \mathrm{~m}$ \\
\hline Carrier frequency & $2 \mathrm{GHz}$ \\
\hline Thermal noise (No) & $10^{-7}$ \\
\hline Pathloss exponent $\alpha$ ) & 2 \\
\hline Maximum transmitted power by UEs & 0.2 watt \\
\hline Maximum transmitted power by relays & 1 watt \\
\hline Resource block bandwidth & $180 \mathrm{KHz}$ \\
\hline
\end{tabular}


Figure (3) shows that the data rate increased by increasing the resource blocks (More resources More data rate). Also the data rate increased by increasing the $\mathrm{D} 2 \mathrm{D}$ pairs for the same number of resource blocks.

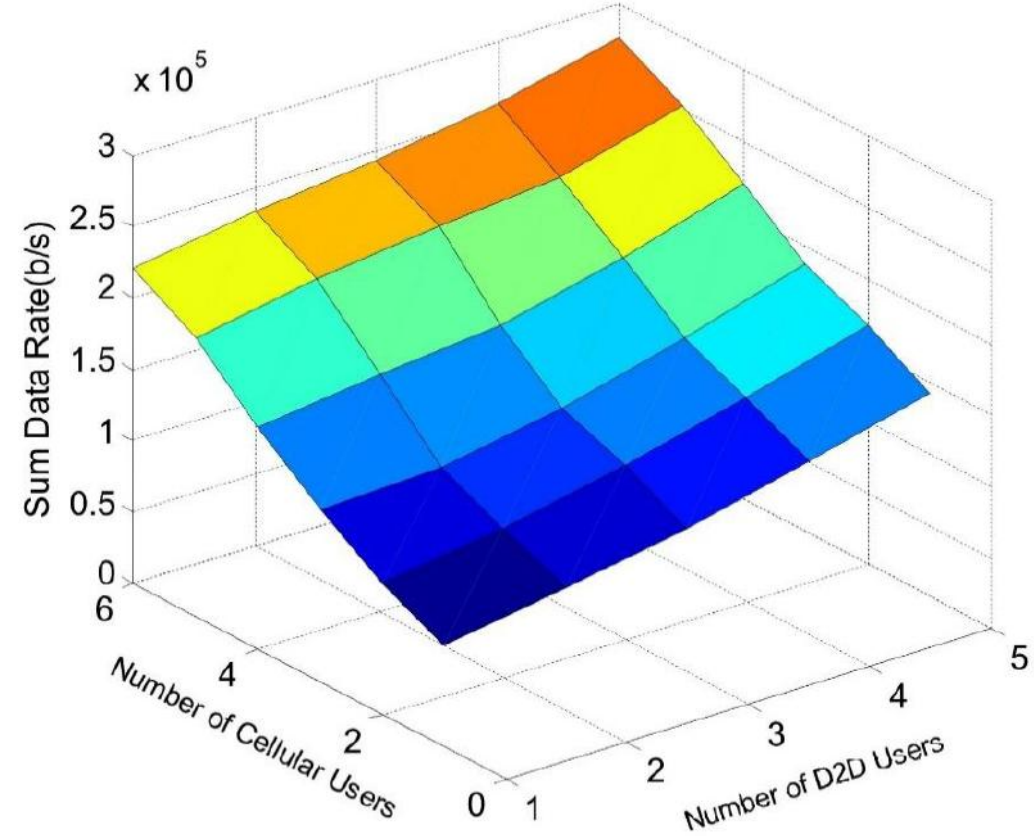

Figure3. System Data Rate Compared to Number of D2D Users and Number of Resource Blocks (Number of Cellular Users).

In fact solving the allocation problem centrally is very complex, while auction algorithm provides near to the optimal solution using distributed manner with very low complexity. The system data rate for different number of D2D users and cellular users is plotted as shown in Figure (4) and Figure (5).

The difference between data rate obtained by optimal and auction based allocation is small. The system data rate using auction is also compared to random allocation and it is observed that the auction provided better data rate than random allocation.

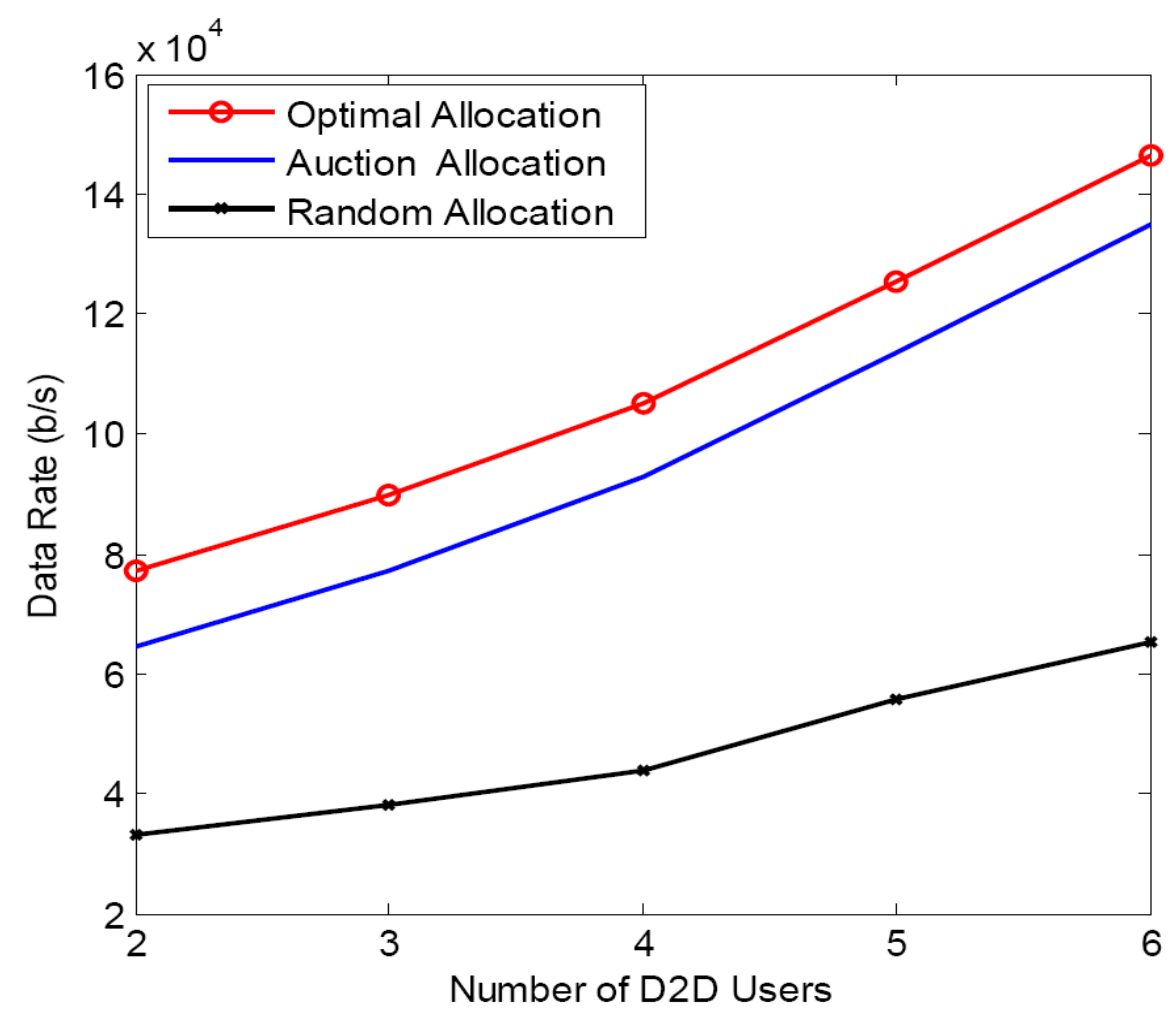

Figure4. Comparison of Auction Algorithm with Optimal and Random Allocation (2 cellular UEs). 


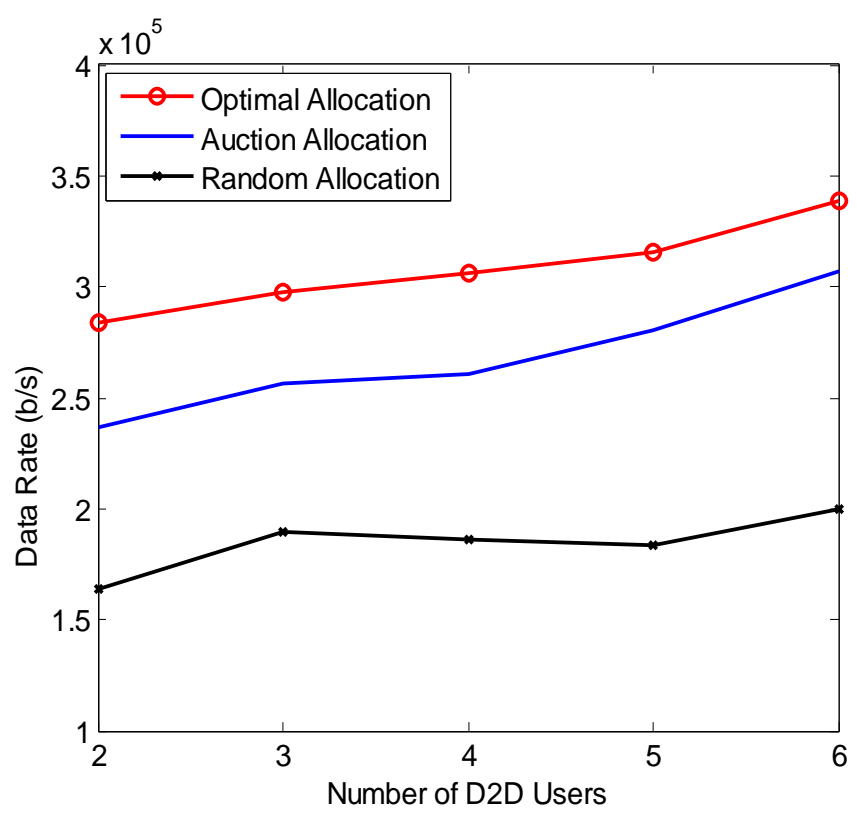

Figure5. Comparison of Auction Algorithm with Optimal and Random Allocation (6 cellular UEs).

The optimal solution is obtained using summing the maximum $\mathrm{N}$ entries of the $\mathrm{R}$ (data rate) matrix given in equation (13). The minimum efficiency obtained by auction method is 0.82 as shown in figure (6), where the efficiency is calculated as $\eta=\frac{\Re_{\text {optimal }}}{\Re_{\text {Auction }}}$.

The method used to solve for optimal allocation does not consider the fairness of assignment while the auction make D2D user share resource with cellular users fairly.

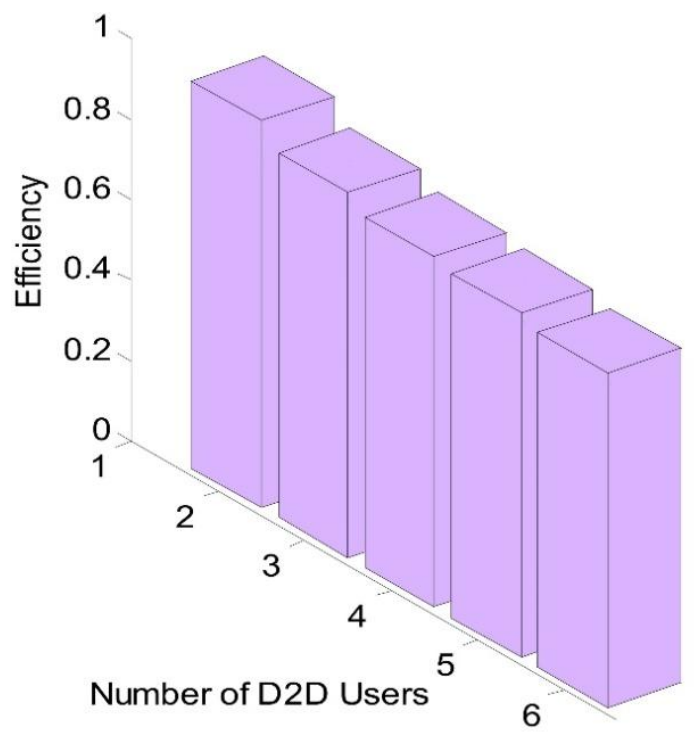

Figure6. Efficiency of Auction based Allocation

\section{Conclusions}

The resource allocation problem is formulated to increase the spectral efficiency and the system data rate. Then, a low complexity distributed algorithm is introduced to solve the resource allocation problem in multitier heterogeneous cellular network with D2D communication underlaying macro users.

The co-channel interference is considered while the D2D users share the same resources with cellular users. The simulation results show that, the auction-based algorithm provide near to optimal solution with minimum efficiency equal to 0.82 with very low complexity. The data rate is increased by increasing the number of resource blocks. The data rate also increased by increasing the D2D pairs for the same number of Resource blocks. The auction algorithm also share the resources fairly. 
The auction process in each relay converges in relatively small number of iterations even in dense scenarios. In the future work, the energy efficiency will be considered besides the spectral efficiency. Also, different auction types will be used to obtain higher efficiency.

\section{REFERENCES}

[1] Chen Xu, Lingyang Song, and Zhu Han, "Resource Management for Device-to-Device Underlay Communication," Springer, 2014.

[2] Klaus Doppler, Mika Rinne, Carl Wijting, Cássio B. Ribeiro, and Klaus Hugl, "Device-to-Device Communication as an Underlay to LTE-Advanced Networks," IEEE Communications Magazine,VOL. 47, NO. 12, pp. 42-49, 2009.

[3] Lei Lei and ZhangduiZhong, "Operator Controlled Device-to-Device Communication in LTEAdvanced Networks," IEEE Wireless Communications, VOL. 19, NO. 3, pp. 1536-1284, 2012.

[4] Gábor Fodor, Erik Dahlman, Gunnar Mildh, Stefan Parkvall, Norbert Reider, GyörgyMiklós,andZoltánTurányi,"Design Aspects of Network Assisted Device-to-Device Communications," IEEE Communications Magazine, pp. 170 - 177, 2012.

[5] Chia-Hao Yu, Olav Tirkkonen, Klaus Doppler and Cassio Ribeiro, "Power optimization of device-to-device communication underlaying cellular communication," IEEE International Conference on Communications, Dresden, pp. 1-5, 2009.

[6] Tao Peng, Qianxi Lu, Haiming Wang, Shaoyi Xu and Wenbo Wang, "Interference Avoidance Mechanisms in the Hybrid Cellular and Device-to-Device Systems," IEEE 20th International Symposium on Personal, Indoor and Mobile Radio Communications, Tokyo, pp. 617-621, 2009.

[7] Mohammad Zulhasnine, Changcheng Huang, and Anand Srinivasan "Efficient Resource Allocation for Device-to-Device Communication Underlaying LTE Network," IEEE 6th International Conference on Wireless and Mobile Computing, Networking and Communications, pp. 368 - 375, 2010.

[8] Furqan Ahmed, Alexis A. Dowhuszko, and Olav Tirkkonen, "Distributed Algorithm for Downlink Resource Allocation in Multicarrier Small Cell Networks", International Workshop on Small Cell Wireless Networks, pp. 6802 - 6808, 2012.

[9] Chen Xu, Lingyang Song, Zhu Han, Dou Li, and Bingli Jiao, "Resource Allocation Using A Reverse Iterative Combinatorial Auction for Device-to-Device Underlay Cellular Networks," Globecom 2012 - Wireless Communications Symposium, pp. 4542-4547, 2012.

[10] Chen Xu, Lingyang Song, Zhu Han, Qun Zhao, Xiaoli Wang, and Bingli Jiao, "InterferenceAware Resource Allocation for Device-to-Device Communications as an Underlay Using Sequential Second Price Auction," IEEE ICC 2012 Ad-hoc and Sensor Networking Symposium, pp. 445-449, 2012.

[11] Chen Xu and Lingyang Song, "Efficiency Resource Allocation for Device-to-Device Underlay Communication Systems: A Reverse Iterative Combinatorial Auction Based Approach," IEEE JOURNAL ON SELECTED AREAS IN COMMUNICATIONS/ SUPPLEMENT, VOL. 31, NO. 9, pp. 348-358, 2013.

[12] Bernd Holfeld, Tobias Jaeuthe and Thomas Wirth, "Resource Sharing with Minimum QoS Requirements for D2D Links Underlaying Cellular Networks", IEEE 81st Vehicular Technology Conference (VTC Spring), pp. 1-7, 2015.

[13] Ehab H. Abdelhay, Fayez W. Zaki, Sherif S. Kishk and Hossam S. Moustafa, "Synchronous Direct and Multi-hop Transmission in Multi-hop Uplink LTE-A using AMMCS ", International Journal of Review in Electronics and Communication Engineering, Vol. 3, No. 6, 2015.

[14] N. H. Almofari,S. Kishk, andF. W. Zak, "Auction Based Algorithm for Distributed Resource Allocation in Multitier-Heterogeneous Cellular Networks," $11_{\text {th }}$ international Conference on Computer Engineering \& Systems (ICCES), Cairo, Egypt, pp. 426-433, 2016.

[15] D. P. Bertsekas, "Auction Algorithms for Network Flow Problems: A Tutorial Introduction," Lids-P, pp. 1-54, 1992

[16] Ehab H. Abdelhy, Fayez W Zak, Sherif S. Kishk, "C2. Uplink Performance of MIMO Multi-hop LTE-A Based on Link Adaptation," in 32 ${ }^{\text {nd }}$ National Radio Science Conference, pp. 329-339, 2015. 